\title{
Quality Indicators of Blood Utilization in a Tertiary Care Centre in the North-Eastern India
}

\author{
Dr.Kh. Memtombi Devi ${ }^{1}$, Dr. A. Barindra Sharma ${ }^{2}$, Dr.L.Dorendro Singh ${ }^{3}$, \\ Dr.Ksh.Vijayanta ${ }^{4}$, Dr.S.T.Lalhriatpuii ${ }^{5}$, Dr.A.Meina Singh ${ }^{6}$ \\ 1,3,4,5 Post-graduate trainees, ${ }^{2}$ Associate Professor, ${ }^{6}$ Professor and Head \\ Department of Immunohaematology \& Blood Transfusion, Regional Institute of Medical Sciences, \\ Imphal,Manipur, India
}

\begin{abstract}
Blood transfusion has become an essential part of patient management in modern medicine. Blood is a scarce resource and the blood needs still exeed its supply. Blood and blood components are frequently ordered and utilized without proper analysis of the real needs. Inappropriate ordering and use of blood can burden the physical and human resources of a health-care facility and increase the cost of medical care. Various strategies and quality indicators have been developed to monitor the justification of appropriateness and efficiency of transfusion therapy. The study was taken up to evaluate the utilization of blood and status of transfusion practice in a tertiary teaching hospital in the North-Eastern India. The quality indicators analysed were Cross-match to Transfusion ratio(C: T), Transfusion index(TI), Transfusion probability(\%T), RBC-unit expiration rate, $R B C$-unit wastage rate and RBC concentrate (packed RBC) to Whole blood ratio( PRBC: WB). The results were within the acceptable limits as per other studies available in the literature. But the PRBC: WB ratio was lower than the WHO recommendation of 90: 10. Continuous monitoring will improve the blood transfusion services and will contribute towards shortening of demand- supply gap.
\end{abstract}

Key words: Blood transfusion, Blood utilization, Cross-match, Quality indicators, Transfusion practice

\section{Introduction}

The transfusion of blood and blood components has become an integral part of patient management in modern healthcare. In developing countries like India, there is scarcity of voluntary blood donors leading to insufficient blood supply. The blood needs still exeed its supply. To overcome this, the performance of Blood Transfusion Services(BTS) can be increased by increasing the level of resources used in the collection and production of blood components or by utilizing existing resources more efficiently.[1 ].The BTS can reach the optimum level of efficiency in terms of quantity and quality of blood and blood components through the implementation of a quality management system in all phases of the collection, processing and storage of the blood.[1 ].The American Association of Blood Banks(AABB) defines quality indicators as the specific performance measurements designed to monitor one or more processes during a defined time and are useful for evaluating service demands, production, adequacy of personnel, inventory control, and process stability.[2 ] . Blood and blood components are frequently ordered without proper analysis of the real needs. Such practices have greater implications in resource-constrained settings.[3]. Injudicious over-ordering of blood can burden the physical and human resources of a health care facility and increase the cost of medical care.[3 ]. Data from many developing countries have shown gross over-ordering of blood in $40 \%$ to $70 \%$ of patients transfused.[4,5] The objective of the study was to evaluate the utilization of blood and the status of transfusion practices in Regional Institute of Medical Sciences Hospital, Imphal, a tertiary care centre in the North-eastern India.

\section{Material And Methods}

The retrospective and cross-sectional study was conducted in the Department of Immunohaematology and Blood Transfusion, Regional Institute of Medical Sciences Hospital, Imphal over a period of one year from September 2012 to August 2013. The records of all the patients, daily blood collection, cross-matching, actual transfusion and blood stock were taken. The parameters used in the analysis were:

1.Cross-match to transfusion ratio(C: T): No. of cross-matched RBC units/ No. of transfused RBC units

2.Transfusion index (TI): The number of RBC units transfused relative to the number of cross-matched units, calculated as; No. of transfused RBC units/ No. of cross-matched RBC units

3.Transfusion probability(\% T): No. of transfused RBC units/ No. of cross-matched RBC units x 100

4.RBC unit expiration rate(expired unit- an RBC unit that was discarded because of its lifespan exceeding allowable time for transfusion i.e. outdated) : No. of expired RBC units/ ( No. of transfused RBC units+ No. of expired RBC units) x 100 
5.RBC unit wastage rate( wasted unit - an RBC unit that was discarded before expiration because of haemolysis, discolouration, breakage, clots, underweight, overweight, return of unused unit exceeding allowable time limits etc.): No. of wasted RBC units/ (No. of transfused RBC units+ No. of wasted RBC units) x100

6.RBC concentrate(Packed RBC) transfused to Whole blood transfused ratio(PRBC: WB): No. of units used as $\mathrm{PRBC} /$ No. of units used as WB

The transfusion transmissible infection positive cases were not included in the calculation.

\section{Results}

The total blood collection during the period was 11,243 units, of which the voluntary blood donors constituted $46.12 \%$ and the replacement blood donors, $53.88 \%$. The total whole blood and blood components issued were 13,053. The total cross-matched RBC units were 10,101 and the total RBC units transfused were 9821. A total of 48 RBC containing units were discarded including 20 expired units and 28 wasted units. The C: $\mathrm{T}$ ratio was found to be 1.02 and TI was 0.97 . The transfusion probability $(\% \mathrm{~T})$ was calculated as $97.2 \%$. The $\mathrm{RBC}$ - unit expiration rate was $0.2 \%$ and the RBC-unit wastage rate was $0.28 \%$. The PRBC : WB ratio was 5.34 ( $53.4: 10)$.

\section{Discussion}

Blood transfusion is considered appropriate when it is used to treat conditions leading to significant morbidity and mortality, and which cannot be prevented or managed effectively by other means.Blood is an expensive and scarce resource. Unnecessary transfusions may cause a shortage of blood products for patients in real need.[6]. Various strategies have been developed to reduce the inappropriate use of blood and blood components. These include guidelines as well as monitoring of transfusion practice, education and self audit by clinicians.[7 ]. Continuous monitoring will help to supply safe blood and blood products to needy patients and prevent over-utilization of a scarce resource. Various quality indicators have been identified. As per WHO- the working definition of an indicator is 'a variable with characteristics of quality, quantity and time, used to measure changes in health and health-related situation, directly or indirectly, the progress made in addressing it and providing a basis for developing adequate plan for improvement '. [ 8 ]. The role of quality in transfusion practice is looked at three contexts: the blood collection centre, the transfusion service and clinical practice. All indicators have a part to play in maintaining quality, monitoring performance and ensuring safety and integrity for the recipient, donor and staff.[8 ].

The C: T ratio is often used as a measure of the efficiency of blood ordering practice. The desirable C: $\mathrm{T}$ ratio varied from 2 to 3 in different studies.[9,10,11]. The value of 1.02 in our study is within the desirable limits. Kozarzewska $\mathrm{M}$ et al have reported the values justifying blood ordering and utilization as $\mathrm{C}: \mathrm{T}$ ratio < or=3, \% T $>$ or=30 and TI $>$ or=0.5.[12]. The TI is an average number of units used per patient cross-matched and determines whether the number of units ordered is appropriate. [12]. In the same study, the findings of TI were $0.42,0.15$ and 0.058 respectively in three different groups of patients which indicated that only in the first group the blood ordering was acceptable.It has also been reported that when TI $=0.5$ means significant blood utilization.[13]. The transfusion probability $(\% \mathrm{~T})$ also varied as $18.8 \%$ in the $1^{\text {st }}$ group, $8.69 \%$ in the $2^{\text {nd }}$ group and $2.94 \%$ in the $3^{\text {rd }}$ group which suggested to change the perioperative blood ordering policy.[12]. The $\% \mathrm{~T}$ of 97.2 in the present study is significant.

In another study by Novis DA et al in different hospitals of the United States, the C:T ratios were 1.5 or less, red cell expiration rates were $0.1 \%$ or less and red cell unit wastage rates were $0.1 \%$ or less in the top performing institutions. The bottom performing participants had $\mathrm{C}$ : $\mathrm{T}$ ratios above 2.0, $\mathrm{RBC}$ unit wastage rate as $3 \%$ and RBC unit expiration rate above 5\%.[14]. In our study, the RBC unit expiration rate was $0.2 \%$ and the $\mathrm{RBC}$ unit wastage rate was $0.28 \%$ which are close to the top performing groups. Morish $\mathrm{M}$ et al also have found the rate of discard for whole blood as $3.7 \%$ and for the packed RBC as 0.6.[1].

The PRBC : WB ratio of $53.4: 10$ in the present study is less than the WHO recommendation of 90: 10.

\section{Conclusion}

Blood is a scarce resource. The demand is always more than the supply. Blood and blood components are frequently ordered and utilized in many hospitals without proper analysis of the real needs, deviating from evidence-based practice. Continuous monitoring through standard quality indicators of a quality management system will improve the blood transfusion services. Appropriate ordering and use of blood and blood components will contribute towards filling up of the gap between demand and supply. 


\section{References}

[1] Morish M, Ayob Y, Naim N, Salman H, Muhamad NA, Yusaff NM. Quality indicators for discarding blood in the National Blood Center, Kuala Lumpur. Asian J Transfus Sci 2012 ; 6(1): 19-23

[2] Roback JD, Grossman BJ, Harris T,Hillyer CD. Ed. Technical Manual, 17 $7^{\text {th }}$ edition, American Association of Blood Banks,2011.Bethesda, Maryland.p.24

[3] Chawla T, Kakepoto GN, Khan MA. An Audit of Blood Cross-match ordering practices at the Aga Khan University Hospital: First step towards a Maximum Surgical Blood ordering Schedule . J Pak Med Assoc 2001; 51 : 251-54

[4] Lowery TA, Clark JA. Successful implementation of Maximum Surgical Blood order Schedule. J Med Assoc, Ga 1989; 79: 15558

[5] Hardy NM, Bolen FH, Shatney CH. Maximum surgical blood order schedule reduces hospital costs . Am Surg 1987; 53: 223-25

[6] The Clinical Use of Blood Handbook, World Health Organization, Blood Transfusion Safety, Geneva,2002.p.3-4.

[7] Hui CH,William I, Davis K. Clinical audit of the use of fresh-frozen plasma and platelets in a tertiary teaching hospital and the impact of a new transfusion request form. Internal Medicine Journal 2005; 35(5): 283-88

[8] Safe blood and blood products: indicators and quality of care. World Health Organization, Regional office for the western Pacific, Manila, Philippines. October 1996

[9] Friedman BA, Oberman HA, Chadwick AR. The maximum surgical blood order schedule and surgical blood use in the United States. Transfusion 1976; 16: 380-87

[10] Palmer T, Wahr JA, O’ Reilly, Greenfield ML. Reducing unnecessary cross-matching : a patient-specific blood ordering system is more accurate in predicting who will receive a blood transfusion than the maximum blood ordering system. Anesth Analg 2003; 96 : 369-75

[11] Mead JH, Anthony CD, Sattler M. Hemotherapy in selective surgery: an incidence report, review of literature and alternatives for guideline appraisal. Am J Clin Path 1980; 74: 223-27

[12] Kozarzewska M, Mackowiak M, Steler J, Krefta M, Hasak L, Kardel-Reszkiewicz E. The analysis of blood order protocol. Anaesthesiology Intensive Therapy 2011; XLIII(2): 61-63

[13] Vibhute M, Kamath SK, Shetty A. Blood utilization in elective general surgery cases: requirements, ordering and transfusion practices. J Postgrad Med 2000; 46: 13-17

[14] Novis DA, Renner S, Friedberg R, Walsh MK, Saladino AJ. Quality indicators of blood utilization Three College of American Pathologists Q-Probes Studies of 12288404 Red Blood Cell Units in 1639 Hospitals. Arch Pathol Lab Med 2002; 126 : $150-56$ 